\title{
Jitter-induced Max-of-N fluence distribution at the National Ignition Facility
}

\author{
Zhi M. Liao $\odot$ * Christopher W. Carr, Giuliana Pallotta, \\ Wade Williams, and Paul Wegner \\ Lawrence Livermore National Laboratory, Livermore, California, United States
}

\begin{abstract}
Max-of-N fluence is the maximum peak fluence at a given location over $N$ number of shots and is important for calculating fluence dependence for intrinsic laser-induced optic damage. Previously, we observed the Max-of-N effect on the National Ignition Facility and developed an ad-hoc model to calculate its effect. In this work, we attempt to understand the fundamental mechanism that causes this Max-of-N effect. We conclude that the primary fundamental mechanism responsible for this effect is dominated by the combination of fluence variations and pointing jitter of the laser. This discovery both strengthens our model for predicting optics longevity and gives us insight into how to mitigate this effect. (C) The Authors. Published by SPIE under a Creative Commons Attribution 4.0 Unported License. Distribution or reproduction of this work in whole or in part requires full attribution of the original publication, including its DOI. [DOI: 10 .1117/1.OE.60.3.031006]
\end{abstract}

Keywords: optics; laser damage; laser beam characterization; statistical optics.

Paper 20200913SS received Jul. 29, 2020; accepted for publication Nov. 2, 2020; published online Nov. 23, 2020.

\section{Introduction}

Control of the onset of damage on modern optical surfaces has improved so significantly ${ }^{1-3}$ that the damage that occurs on the National Ignition Facility (NIF)'s fused silica optics surfaces is now largely dominated by in-situ contaminations. ${ }^{4,5}$ The fluence and shot dependence of these damage mechanisms and efforts to eliminate them are discussed elsewhere. ${ }^{4,5}$ As these sources of contamination are removed, operating limits are still be constrained by the limits of pristine surfaces. The inherent optics lifetime (endurance of an optic before it needs to be repaired or replaced) of a pristine surface is governed by the population of damage precursors residual to fabrication and fluence distribution of the laser. This is simple if there is one shot. However, as optics are exposed to repeated laser exposure, factors such as random fluence fluctuations and beam jitter can complicate the calculation. ${ }^{6}$ Although Melninkaitis et al. ${ }^{6}$ were interested in how these factors impact laser damage threshold measurements such as S-on-1, these same factors also impact how damage is initiated with multiple exposures. Max-of-N fluence distribution is the maximum peak fluence over $N$ number of shots and was developed ${ }^{7}$ to explicitly address the effect of repeated laser exposure to optics. Although the mean and the max fluence of each shot can remain constant at each location, random fluence fluctuations can drive up the maximum fluence that each location will be exposed. As a result, the Max-of-N effect is the change of the fluence distribution as a function of repeated, constant-energy laser exposure. If no fraction of a beam incident on an optic is of sufficient fluence to initiate any of the damage precursors, understanding the Max-of-N fluence is unnecessary. Conversely, if all areas of the beam exceed the fluence necessary to initiate damage in all precursors, then there is no Max-of-N effect; the first laser shot initiates all of the damage sites, and there is no increase of damage sites with additional shots provided there is no damage fatigue phenomenon. In all other cases, regardless of laser architecture, the Max-of-N fluence distribution is critical in balancing how high the laser should run and the intrinsic damage limit that is posed by the optics (which drives the optics cost). In this work, we attempt to understand the fundamental mechanism that drives this Max-of-N effect on NIF and, by extension, on other large lasers. We are aware that, although pointing jitter is a universal phenomenon that all lasers must deal with, it could very well be that other high energy

*Address all correspondence to Zhi M. Liao, zman@llnl.gov 
(HE) laser systems have other mechanisms that dominate the Max-of-N effect. Nevertheless, our empirical formalism for calculating the Max-of-N fluence distribution and our approach to connecting the physical mechanism to the observed effect would provide a beneficiary foundation for all other high-energy lasers systems.

The inherent optics lifetime limitations imposed by these precursors are quantified by the fluence-dependent damage density, $\rho(\phi)$, typically obtained from small-aperture test stations. ${ }^{8}$ To apply these measurements to NIF optics, the number of initiations $\left(N_{\text {init }}\right.$ per optic) is calculated given the laser fluence probability density function (PDF), $f(\phi)$, and the empirically-derived fluence-dependent damage density, $\rho(\phi)$, as follows:

$$
N_{\text {init }}=A \cdot \int \rho(\phi) \cdot f(\phi) \cdot \mathrm{d} \phi,
$$

where $A$ is the area of the optic. ${ }^{2}$ Because various pulse shapes are routinely used for different purposes, the pulse shape dependence is taken into consideration by scaling the fluence with respect to pulse shape ${ }^{9}$ to account for the effect of thermal diffusion in heating up the damage precursors. As a result, $\phi$ used for initiation calculations is the damage equivalent fluence (e.g., equivalent to a 3-ns Gaussian pulse shape). This is straightforward for a single shot, where we assume that the fluence distribution is Gaussian with mean $\mu$ and standard deviation $\sigma_{s}$ as follows:

$$
f_{S}\left(\phi ; \mu, \sigma_{S}\right)=\frac{1}{\sigma_{S} \sqrt{2 \pi}} e^{-\frac{(\phi-\mu)^{2}}{2 \sigma_{S}^{2}}}
$$

As an optic accumulates additional exposures, the maximum fluence that each local space sees evolves from the effects of shot-to-shot contrast, i.e., local fluence variation over shots. Depending on the extent of the shot-to-shot variations in the spatial fluence distributions, the Max-of-N fluence exposure on the optic surface can become significantly higher. Figure 1 shows two series of shot sequences on NIF, one with 16 shots at HE and one with 12 shots at lower energy (LE) measured at two different locations of the same laser, at the fundamental wavelength of $1053 \mathrm{~nm}$ (denoted $1 \omega$ ) and after converting frequency via potassium dihydrogen phosphate crystal KDP to $351 \mathrm{~nm}$ (denoted $3 \omega$ ). Although individual shots (denoted in Fig. 1 as shot) are delivering the same energy (i.e., mean fluence), the mean of Max-of-N fluence distribution as measured (denoted in Fig. 1 as MaxN) is increasing as a function of shot number. Without the Max-of-N effect, we should see no difference in single shot and repeated shot versus shot number. As a result, the number of initiations also increases, effectively reducing the optic's lifetime. One way to visualize this effect is to imagine the hot spot (i.e., local fluence maxima) of the laser beam moving without constraints to different parts of the beam so that with enough shots it

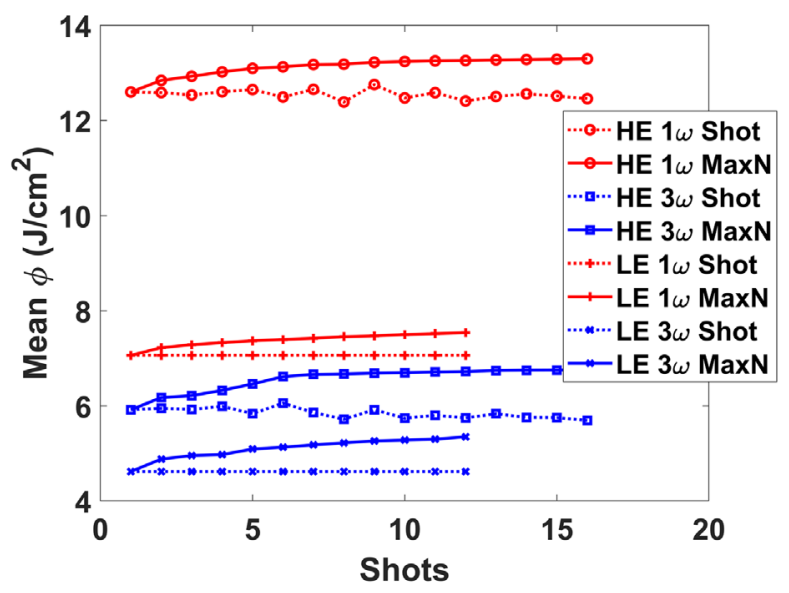

Fig. 1 Measured single-shot and Max-of-N (MaxN) mean fluence for four series of shot sequences with different energy levels (HE, LE) and at different sections of the laser $(1 \omega, 3 \omega)$. 
eventually covers much of the optic surface. As a result, the maximum fluence seen on each location on the optic is substantially raised. From the optic's defect precursor point of view, these "moving" hot spots increase the likelihood that the higher fluence coincides with the location of precursors, therefore, increasing the number of initiated damage sites with shot number.

To account for multiple shots on the same optic, the single-shot fluence distribution is replaced by the Max-of-N fluence distribution $f_{S S \max }(\phi, N)$, i.e., maximal local fluence that an optic has been exposed to over $N$ number of shots. The observed Max-of-N fluence distribution on $\mathrm{NIF}^{10,11}$ has been empirically modeled as having the same single-shot fluence distribution except with a shot-dependent mean fluence $\mu_{\max }$.

$$
f_{S S \max }\left(\varphi ; N, \mu, \sigma_{S}, \sigma_{T}\right)=f_{S}\left(\varphi ; \mu_{\max }\left(N, \mu, \sigma_{T}\right), \sigma_{S}\right)
$$

where $\mu_{\max }$ is the Max-of-N mean fluence and is given as

$$
\mu_{\max }\left(N, \mu, \sigma_{T}\right)=\int x \cdot f_{N}\left(x ; \mu, \sigma_{T}, N\right) \mathrm{d} x,
$$

with $f_{N}$ representing the PDF of the maximum order statistic, ${ }^{12}$ which is written as follows:

$$
f_{N}\left(x ; \phi, \sigma_{T}, N\right)=N \cdot f_{T}\left(x ; \phi, \sigma_{T}\right) \cdot F_{T}\left(x ; \phi, \sigma_{T}\right)^{N-1},
$$

with $f_{T}$ and $F_{T}$ being the PDF and CDF (cumulative density function) of the shot-to-shot fluence variation. The key parameter is $\sigma_{T}$, which we call shot-to-shot contrast. We were able to extract this empirical parameter for a series of data collected at NIF in 2007.

The data consist of two consecutive series of shots, one at LE and the other at HE, where the laser operation was deliberately kept as constant as possible. Furthermore, we had measurements of the laser fluence map at the fundamental wavelength $(1 \omega)$ and after the laser frequency was tripled $(3 \omega)$. As a result, four sets of data were collected (see Table 1). Our empirical model ${ }^{10,11}$ was able to successfully reproduce the Max-of-N experimental data, but the extracted parameter $\sigma_{T}$ was different for each data set, revealing shortcomings for the current approach:

1. It required extracting an empirical parameter $\sigma_{T}$ that required the laser to take multiple shots at the same operation parameter (i.e., energy and pulse shape). This is unrealistic given the cost of each shot and the desirable experimental configuration.

2. We also found that this parameter $\sigma_{T}$ changes at different sections of the laser system (i.e., fundamental $1 \omega$ versus frequency tripled $3 \omega$ ).

3. It is not clear if the validity of the current model will persist as shots increase far beyond what the current data covered ( $\sim 10$ to 15 shots), limiting predictive utility.

4. Without understanding the fundamental mechanism, there is no assurance that this parameter would not change from beam to beam or over time; furthermore, there would not be any ability to mitigate this effect, if desired.

Table 1 Operating parameter for four sets of data collected on NIF in 2007 where the laser was running at different energy levels $(E)$ and pulse shapes (with ignition shape and flat-in-time) at two different sections of the laser system $(1 \omega, 3 \omega)$. The extracted parameter $\sigma_{T}$ is the shot-to-shot contrast measured for each data set.

\begin{tabular}{lccr}
\hline \hline Shot series & Pulse shape & $1-\omega$ light & 3- $\omega$ light \\
\hline $\mathrm{HE}$ & $20 \mathrm{~ns}$ & $E=14 \mathrm{~kJ}$ & $E=7.4 \mathrm{~kJ}$ \\
& Ignition-shaped & $\sigma_{T} \sim 3.4 \%$ & $\sigma_{T} \sim 8.5 \%$ \\
$\mathrm{LE}$ & $1 \mathrm{~ns}$ & $E=3 \mathrm{~kJ}$ & $E=2 \mathrm{~kJ}$ \\
& Flat-in-time & $\sigma_{T} \sim 4.2 \%$ & $\sigma_{T} \sim 9.0 \%$ \\
\hline \hline
\end{tabular}


In this work, we investigated the possible mechanism behind the observed Max-of-N effect and addressed the various concerns described above that have limited the confidence in the current model.

\section{Simulation of Jitter-Induced Max-of-N Effect}

Beam jitter is a well understood phenomenon that is often related to far field pointing issues. In our case, we were concerned with the near field maximum fluence distribution as it relates to the Max-of-N effect. We revisited the collection of data that was used to derive the model in our previous work $^{10,11}$ and found that near field data collected on NIF also recorded the center and edge of the beam on the camera charge-coupled device (CCD). NIF beams typically consist of a clear aperture that is $36 \times 36 \mathrm{~cm}^{2}$ with near field measured by 16 -bit, $1024 \times 1024$ pixels. Depending on how the near field is imaged onto the CCD camera, a typical resolution is $\sim 0.4 \mathrm{~mm}$ per pixel. By plotting the beam location over time, it was evident that the beam wanders randomly shot to shot (see Fig. 2). Furthermore, by analyzing the jitter, we found that the amount of jitter follows a normal distribution with a standard deviation of 2.4 pixels, although we would expect the distribution to be truncated at higher values because of the physical constraint of the jitter movement.

Using this information, we simulate how the Max-of-N of a sequence of shots would look if we assumed that every shot is completely identical and the only variation is that we randomly move the center of the beam as measured by the CCD. This is done using a single fluence map and randomly jittering its position using a random number generator (integer movement) given the jitter range from Fig. 2 for $N$ number of shots. Then we calculated the simulated Max-of-N fluence map (i.e., Sim. MaxN) by taking the maximum peak fluence at each point (pixel) across the shot series. It should be noted that NIF is pretty precise in its ability to deliver 1- $\omega$ energy consistently when running at high energies where amplifiers are saturated; however, when running at lower energies or at the 3- $\omega$ plane, the delivered energies do fluctuate shot to shot although the laser set points are the same. In these cases, we normalized the energies for each series of shots to extract the Max-of-N effect from constant repeated exposure. Figure 3 shows the fluence map of the first shot that we used in this simulation [Fig. 3(a)]. It is worth noting that the large circular features are the blocker deployed on NIF to reduce damage growth on existing damage sites. We plot the actual measured Max-of-N fluence map for all 16 shots measured on NIF in Fig. 3(b). The Max-of-N fluence map shows features similar to those in the first shot, but with spatial broadening and saturation at the higher fluences. The results of the simulation of using only 1 fluence map but randomly jittering shot to shot and then combining them to form the Max-of-N fluence map is shown in Fig. 3(d). The simulated Max-of-N [Fig. 3(d)] results show remarkably strong resemblances to the measured results [Fig. 3(b)]. The PDF of the measured and simulated Max-of-N fluence map are also consistent with each other [Fig. 3(c)].

We follow this by applying the same methodology (initial fluence map with jitter range as the input variable) to the associated shots at the converted 3- $\omega$ beam. The result shows that the same
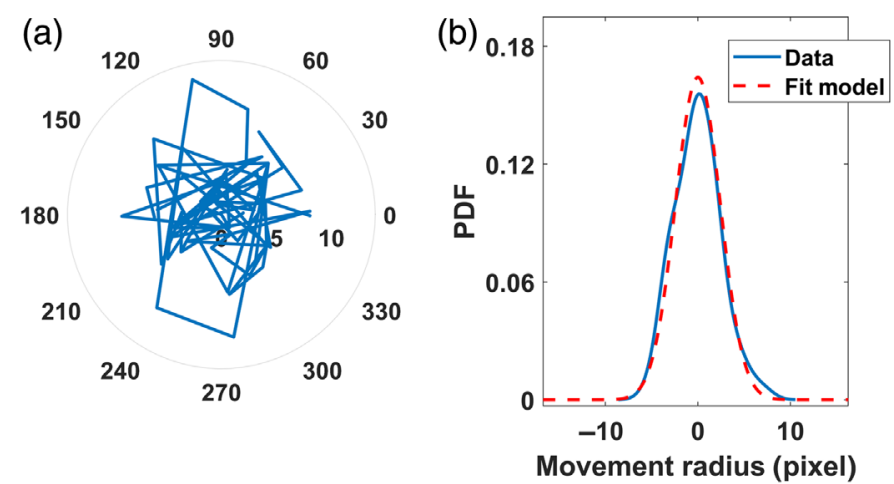

Fig. 2 (a) Polar plot of the pointing jitter of NIF beam over 82 shots on NIF. (b) The PDF of the pointing jitter fitted with a normal distribution with $\sigma=2.43$. Pixel resolution is $\sim 0.4 \mathrm{~mm}$. 

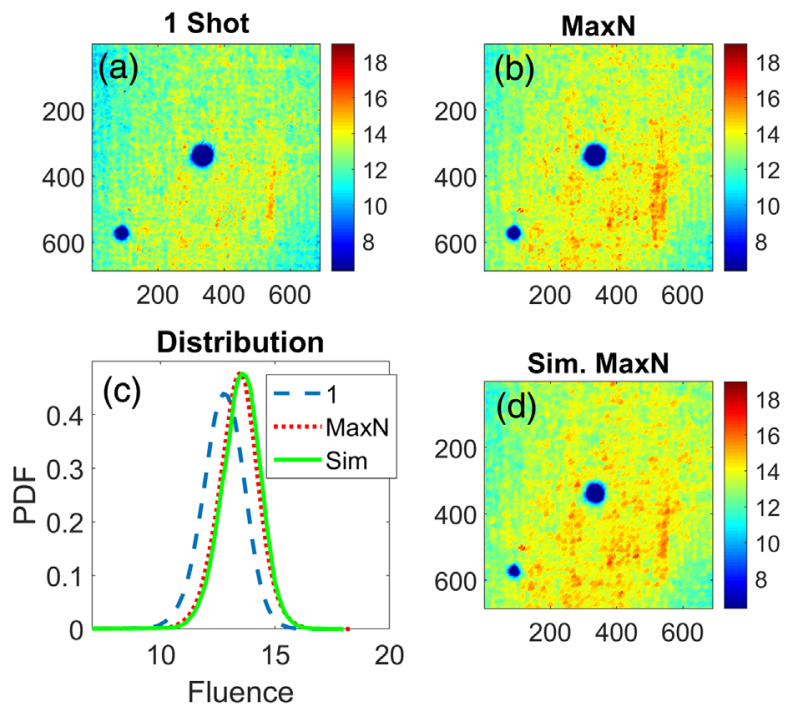

Fig. 3 (a) Measured single-shot fluence map and (b) measured Max-of-N fluence map of 16 shots at NIF operating at HE at the 1- $\omega$ plane. (c) The measured and simulated PDF describing the fluence distribution is plotted. Fluence unit is $\mathrm{J} / \mathrm{cm}^{2}$. (d) Simulation of the Max-of-N fluence distribution using a single fluence map.

trend is also recovered. This is a powerful revelation since, although our empirical model was able to reproduce the same result, we had to use an empirically derived parameter $\left(\sigma_{T}\right)$ from each of the shot series, whereas this result uses only the first shot and a constant parameter describing the beam movement that is the same for the fundamental (Fig. 3) and the 3- $\omega$ converted section of the beam (Fig. 4).

We also applied this methodology to our low-energy shot series, and the same result was reproduced (see Figs. 5 and 6), again with only using a single fluence map from each series and a constant parameter that was derived from the beam jitter data. The fact that the same constant parameter can be used to reproduce the characteristics of a Max-of-N fluence map is a powerful result that eliminates virtually all of the shortcomings of the previous empirical model. In addition, this constant parameter is easily measured for each of the laser beams in NIF.

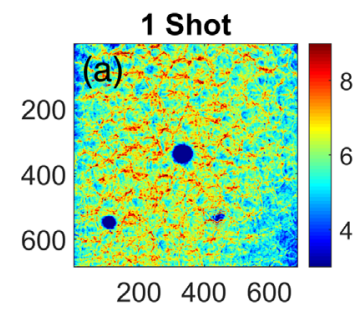

Distribution

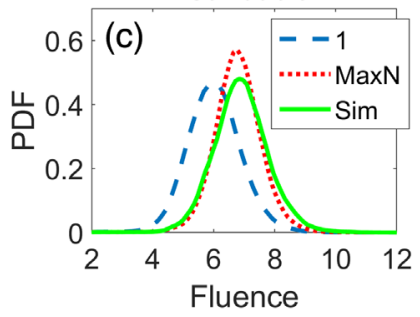

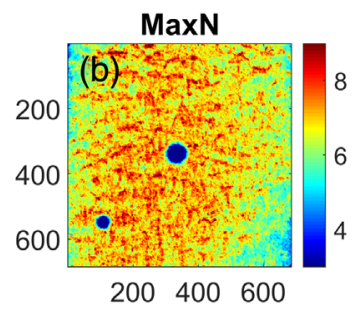

Sim. MaxN

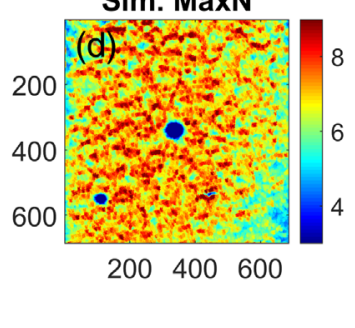

Fig. 4 (a) Measured single-shot fluence map and (b) measured Max-of-N fluence map of 16 shots at NIF operating at $\mathrm{HE}$ at the 3- $\omega$ plane. (c) The measured and simulated PDF describing the fluence distribution is plotted. Fluence unit is $\mathrm{J} / \mathrm{cm}^{2}$. (d) Simulation of the Max-of-N fluence distribution using a single fluence map. 

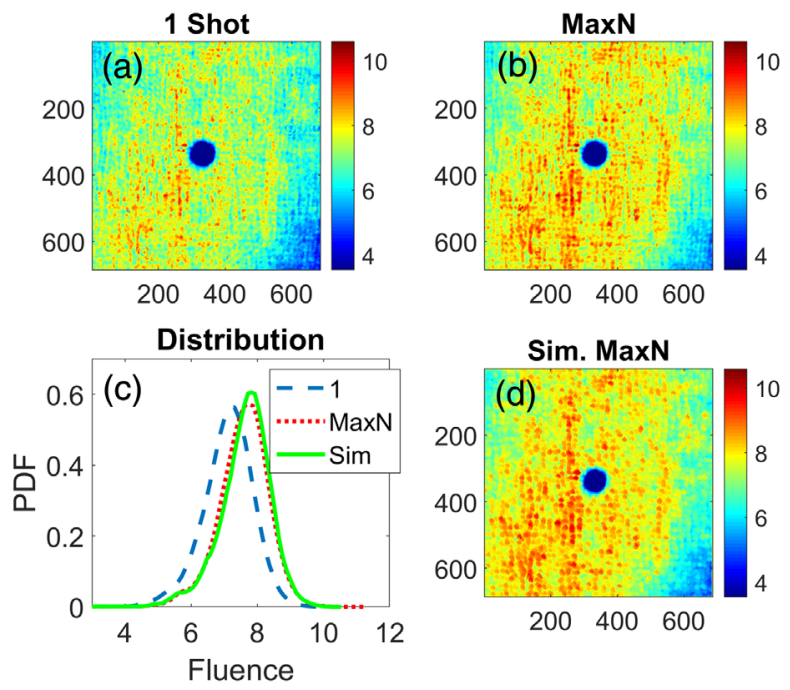

Fig. 5 (a) Measured single-shot fluence map and (b) measured Max-of-N fluence map of 12 shots at NIF operating at LE at the 1- $\omega$ plane. (c) The measured and simulated PDF describing the fluence distribution is plotted. Fluence unit is $\mathrm{J} / \mathrm{cm}^{2}$. (d) Simulation of the Max-of-N fluence distribution using a single fluence map.
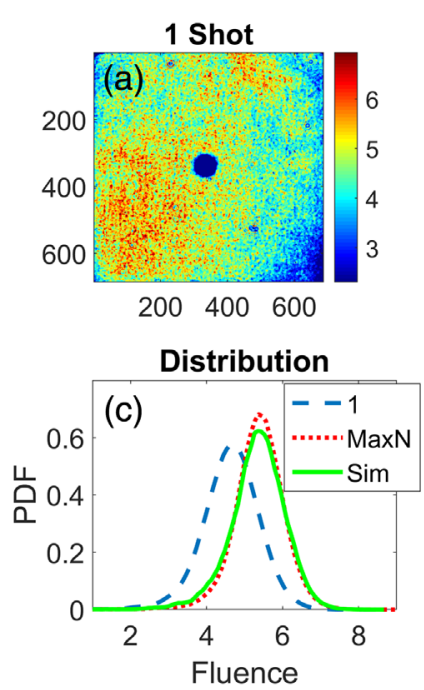
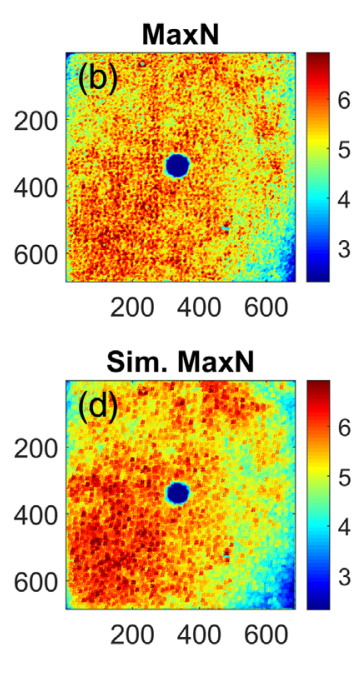

Fig. 6 (a) Measured single-shot fluence map and (b) measured Max-of-N fluence map of 12 shots at NIF operating at LE at the 3- $\omega$ plane. (c) The measured and simulated PDF describing the fluence distribution is plotted. Fluence unit is $\mathrm{J} / \mathrm{cm}^{2}$. (d) Simulation of the Max-of- $\mathrm{N}$ fluence distribution using a single fluence map.

\section{Jitter-Induced Max-of-N Model}

In the last section, we demonstrated that, using a single measured fluence map, we can numerically simulate the effective Max-of-N fluence distribution with only a single constant parameter, the amount of jitter the laser experiences shot to shot. Although this is a very powerful revelation, in practical terms, an analytical solution that can calculate the Max-of-N fluence distribution is still desirable. This is because, currently on NIF, we do not have high-resolution near-field cameras on each of the 192 beamlines. Furthermore, for damage predictions, it is easier to use simple statistical descriptors such as mean and contrast for the numerous types of shots that NIF can support as opposed to trying to scale a canonical fluence map (if it exists) to different energies. In this section, we use our new knowledge of the mechanism that is responsible for the Max-of-N effect to develop an analytical solution that would work for arbitrary shots. 
In theory, we need to calculate the order statistics of elements of the same sample (i.e., the whole fluence map) in which each element might be obtained from a different population (i.e., different local region). Once that is done, the Max-of-N fluence distribution is simply the joint probability distribution of the individual order statistics of various local regions after $N$ shots, which can be, in theory, applied via the Bapat-Beg theorem. ${ }^{13}$ However, the formula to compute the Bapat-Beg theorem is "computationally intractable," 13 but we believe there might be an easier solution that uses our existing empirical model ${ }^{10,11}$ yet leverages our new understanding.

\subsection{Ensemble Statistics}

The fact that using a single measured fluence map can reproduce the maximum fluence distribution of a series of independent shots implies that a single shot's fluence map can reproduce most if not all of the observed Max-of-N effect. In addition, the jitter mechanism also suggests that the local fluence map, i.e., fluence distribution within the jitter range, is the key statistical descriptor. To this end, we image decompose the full fluence map $\Phi$ into an ensemble of smaller local fluence maps, $\Psi_{i}$ (see Fig. 7) as follows:

$$
\Phi=\bigcup_{i=1 \ldots M} \psi_{i}
$$

Each $\Psi_{i}$ is a Gaussian distribution with $\mu_{i}$ and $\sigma_{i}$, and $U$ is the union operator. These statistic descriptors change depending on how finely we divide the square, given the spatial frequency content of the fluence map. We calculate a few interesting values such as the mean of the ensemble contrast $\Sigma$, and the standard deviation (or contrast) of the ensemble mean, $\Gamma$, that can be powerful predictors for the Max-of-N effect.

$$
\begin{gathered}
\Sigma=\frac{\left\langle\sigma_{i}\right\rangle}{\left\langle\mu_{i}\right\rangle}, \\
\Gamma=\frac{\operatorname{std}\left(\mu_{i}\right)}{\left\langle\mu_{i}\right\rangle} .
\end{gathered}
$$

\subsection{Mean of Ensemble Contrast}

The mean of the ensemble contrast $\Sigma$ is the average variation for a specific spatial frequency (or how we finally divide up our fluence map). This value depends on how uniformly distributed the

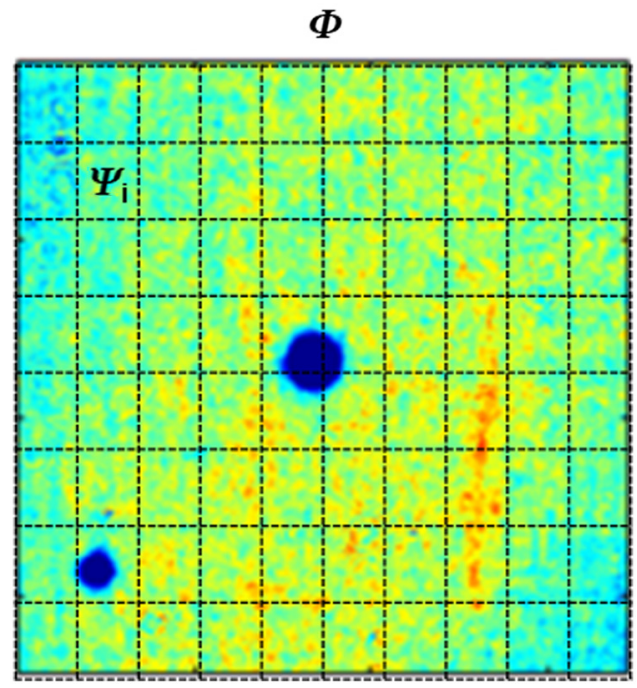

Fig. 7 Single fluence map divided into $N$ ensemble small local fluence maps. 


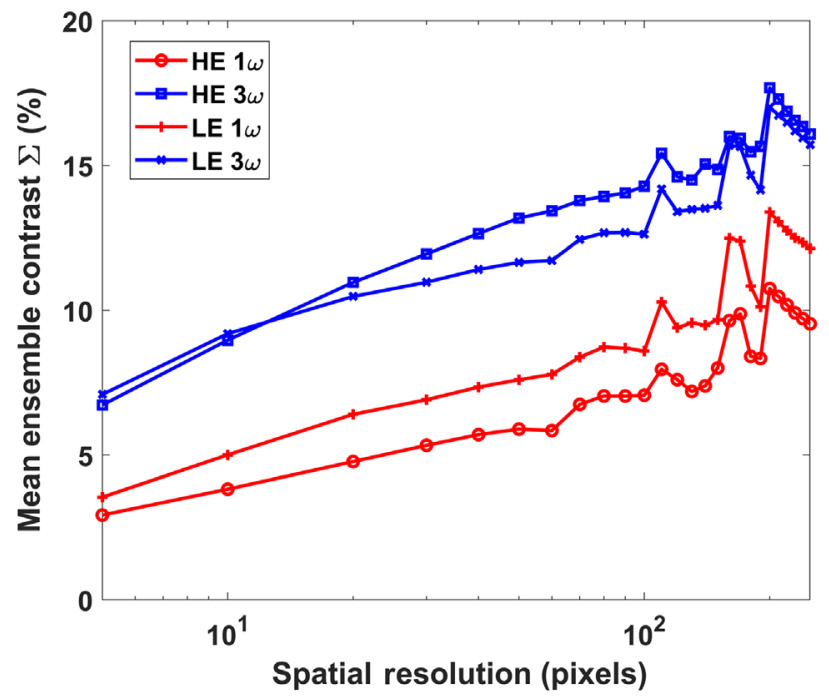

Fig. 8 Calculated ensemble mean contrast versus spatial resolution for the different data series (from fluence map on the first shot of each series). Pixel resolution is $\sim 0.4 \mathrm{~mm}$.

fluences are across the whole laser aperture. If the laser is completely uniform, then we imagine that this value is independent of spatial resolution; however, if there are local hot spots in the beam, this value varies as a function of that spatial frequency but converges to the full beam contrast as the spatial frequency approaches the full dimension of the beam. Figure 8 is plotted below for all four of our data sets using the first shot of each sequence. These results show that the ensemble contrast increases from very high spatial resolution ensembles until it yields to the value of the contrast of the entire beam ( 600 pixels). At the observed jitter range ( $2 \sigma=5$ pixels, see Fig. 2), we found that the mean of the ensemble contrast is exactly the same for the 1- $\omega$ beam (HE 3.4\%, LE 4.2\%) as our derived shot-to-shot contrast $\left(\sigma_{T}\right)$ measured across multiple shots. ${ }^{10}$ However, for the $3-\omega$ beam (HE 7\%, LE 7.5\%), the mean ensemble contrast is about $1.5 \%$ lower than our derived shot-to-shot contrast $\left(\sigma_{T}\right)$ measured across multiple shots. ${ }^{10}$ The difference might be that for the $3-\omega$ beam, although pointing jitter is still the dominate mechanism for the observed shot-to-shot contrast $(\sim 83 \%)$, there is an additional mechanism that contributes to the observed Max-of-N effect. We speculate that this mechanism could be the random fluctuations that come from the nonlinear effect of the frequency conversion system since it occurs only for the 3- $\omega$ beam and not the 1- $\omega$ beam.

$$
\sigma_{\mathrm{T}}=\sum(\text { jitter resolution) }
$$

This should not be surprising since we have already speculated and demonstrated that the beam jitter can reproduce the Max-of-N effect; consequently, what we measure as shot-to-shot contrast is merely the manifestation of the local contrast within the jitter range. The key point is that now we only need a single fluence map measurement to derive this parameter, which is much more practical.

Using the measured value of shot-to-shot contrast extracted from the single fluence map, we were able to reproduce the measured Max-of-N fluence mean for each of the four different data sets using the algorithm developed previously. ${ }^{11}$ The input of the analytical model is individual shot's mean fluence, the output is the calculated Max-of-N fluence, and they agreed with the measured Max-of-N values (Fig. 9).

\subsection{Variation of Ensemble Mean}

The contrast (or standard deviation) of the ensemble mean is a measurement of the spread of the local fluences maps. Again, if the fluence map is completely uniform across it, then the fluence 


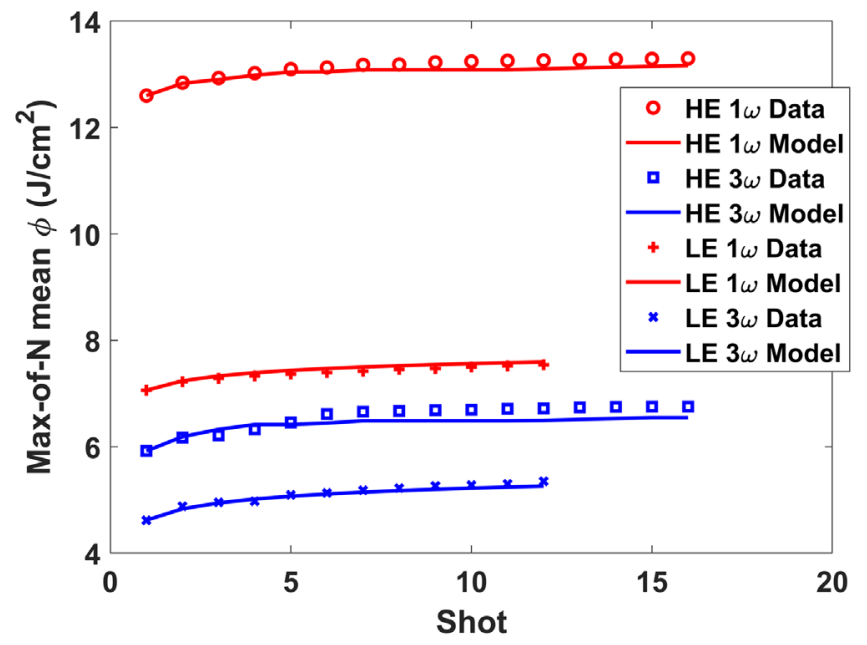

Fig. 9 Measured and model predicted Max-of- $\mathrm{N}$ mean fluence, $\phi$ versus number of shots for the various data sets.

distribution would approach a delta function. However, a measurable ensemble contrast would indicate a difference in fluence distribution across the beam. Take our previous analysis of hot spots moving across the laser beam for example; because of the limit of jitter, the hot spots are limited in terms of how much area it can expose. As shot number increases, each local fluence map would take on the highest local fluence value, and since the same mechanism that yields the rate of increase in the highest fluence of a local fluence also governs the rate of increase in the mean fluence of a local fluence map (i.e., beam jittering,) the contrast of the ensemble mean is the same as the contrast of the ensemble maximum. This implies that the contrast of the ensemble mean is also the asymptotic limit of the Max-of-N contrast (of full beam) as the number of shots becomes large.

$$
\sigma_{\mathrm{MaxN}}=\Gamma(\text { jitter resolution })
$$

Figure 10 shows the contrast of the ensemble mean using the first shot of each of the fourshot series using Eq. (8), and it shows that as jitter increases, which allows the hot spot to expand

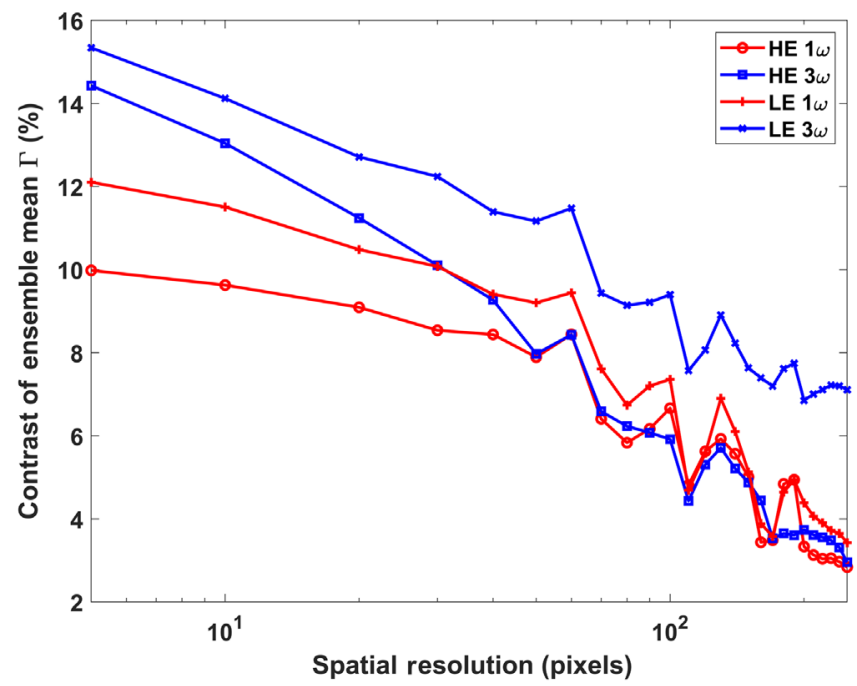

Fig. 10 Calculated contrast of ensemble mean versus spatial resolution for the different data series (from fluence map on the first shot of each series). Pixel resolution is $\sim 0.4 \mathrm{~mm}$. 

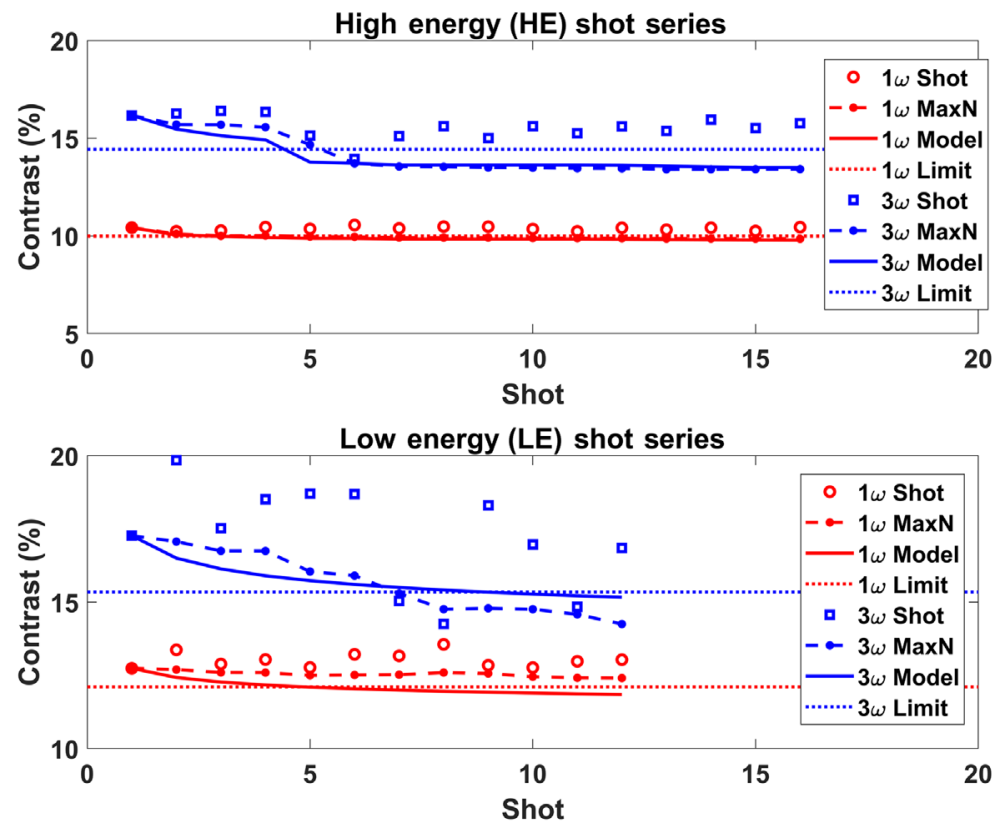

Fig. 11 Measured single-shot and Max-of- $\mathrm{N}$ contrast for the various data series along with the model predicted output and the calculated asymptotic limit.

to an ever-increasing area, the Max-of-N contrast decreases and essentially becomes a delta function for large $N$.

The results of Eq. (8) and Fig. 10 imply that our initial assumption of a constant value contrast $^{8,9}$ [see Eq. (3)] is no longer valid with this understanding. This is because the previous model was empirically derived from shot data without a fundamental mechanism. This is evident in Fig. 11, in which we plotted the contrast of each shot as well as the measured Max-of-N contrast for the four-data series, their HE and LE, and their 1- and 3- $\omega$ locations. Unlike the mean fluence in which our system was able to keep the shot-to-shot value relatively constant (see Fig. 1) for a larger number of shots, the beam contrast has more variation, especially the 3- $\omega$ beam contrast. The only shot sequence in which the beam contrast was kept relatively consistent was the HE 1- $\omega$ series; in this instance, there was a quick drop in Max-of-N measured contrast (1- $\omega \mathrm{MaxN}$ ) (as the average fluence increases) in the first few shots and it asymptotes to the numerically computed $\Gamma$. The other three shot sequences show enough variation of beam contrast that it can be argued that these are no longer similar shots where the same fluence distribution is reproduced while the mathematics of the Max-of-N model was to calculate the maximum fluence of $N$ identical shots. We speculated that the variation in the 3- $\omega$ contrast is due to sensitivity of the frequency conversion, which itself is a nonlinear process that can dramatically alter the beam contrast. The variation in the LE 1- $\omega$ shot series is likely due to lack of gain saturation on the amplifiers that occurred at high energies and helped with stabilizing the fluence contrast.

Since the asymptotic convergence of the single-shot beam contrast to the Max-of-N contrast is so quick, practically speaking, we would assume that, if the laser was stable and able to produce the same contrast every shot, then the asymptotic contrast is $\sim$ the initial contrast for small jitter. This is true for the 1- $\omega$ sequence in which the difference between the average shot contrast and asymptotic contrast is $<1 \%$. For laser systems in which the laser varies significantly in terms of beam contrast, such as the 3- $\omega$ shot sequence where the range of beam contrast is on the order of $5 \%$ or more, then we would either treat each single shot as different energy level shots (i.e., non-constant) or calculate the asymptotic contrast using the minimum of the beam contrast. In essence, in these cases, we no longer have a system that has a constant beam profile (i.e., mean, contrast) that is dominated by beam jitter such as the case for the HE $1 \mathrm{w}$ series; we have a system that has a changing beam profile that is due to either nonlinear frequency conversion or lack of gain saturation. 


\section{Discussion}

Although our results did not substantially change from those obtained by our initial ad-hoc model to calculate the Max-of-N effect, our new ensemble statistic model aids in understanding the fundamental cause of this effect and has diagnostic utility. Specific benefits of the ensemble statistic method include:

1. Extraction of the empirical parameter $\sigma_{T}$ that required the laser to take multiple shots at the same operation point (i.e., energy and pulse shape) is no longer required since we now calculate this parameter using the beam statistics.

2. We have demonstrated that $\sigma_{T}$ may vary with sections of the laser system (i.e., fundamental $1 \omega$ versus frequency tripled $3 \omega$ ). Here this is attributed to the effect of the different spatial contrasts of the fundamental and frequency tripled beam, though spatial filtering could also produce a variance in $\sigma_{T}$.

3. We now calculate the asymptotic limit given our new ensemble statistic model.

4. Understanding that the Max-of-N effect is caused by pointing jitter allows us to track changes to the system and quantify the effectiveness of different mitigation strategies.

We now model the impact on damage initiation due to the Max-of-N effect by changing aspects of the laser such as beam contrast and spatial jitter. The number of initiations versus shot number is calculated using our understanding of the Max-of-N effect using Eq. (1) by simply replacing the single-shot fluence distribution with Max-of-N fluence distribution in Eq. (3). Assuming similar beam characteristics such as our 3- $\omega$ data with mean fluence of $10 \mathrm{~J} / \mathrm{cm}^{2}$ and a beam contrast of $15 \%$ with a jitter of $3 \mathrm{~mm}\left(\sigma_{\mathrm{T}} \sim 8 \%\right)$, we calculate the damage count given a precursor density $\rho(\phi) \sim \phi^{3}$. For our simulation, we vary the fixed beam contrast and the jitter (see Table 2) to illustrate the impact of each parameter. It is worth noting that the shot-to-shot contrast $\left(\sigma_{T}\right)$ for beam contrast of $15 \%$ with different jitter is taken directly from analyzing the fluence map using ensemble statistics [Eq. (9)]. For beam contrast of 5\% and 10\%, since we do not have the associated fluence map readily available, we merely scaled the shot-to-shot contrast at the same spatial frequency at $15 \%$ proportionally to $5 \%$ and $10 \%$, respectively. This seems to be reasonable as the shot-to-shot contrast is merely a local expression of the larger, ensemble fixed-beam contrast. It is worth noting that the numbers presented are for illustration purposes and depend on the functional form of precursor density, ${ }^{3,14}$ and hence the qualitative conclusions will likely vary for different surface preparations. Furthermore, we use the asymptotic Max-of-N contrast for shots $>10$ per Eq. (10) to account for the slight reduction in beam contrast.

Figure 12 shows that changing the beam contrast and jitter can have a dramatic effect on the Max-of-N mean fluence and the damage count. Reducing the fixed beam contrast or the jitter slows the rate of increase for the Max-of-N beam fluence and, as a result, the number of initiations. For example, suppose an optic's lifetime is determined when damage count exceeds 20 . By reducing the beam contrast from $15 \%$ to $10 \%$ to $5 \%$ while keeping the jitter constant, the number of shots that an optic can survive increases from five shots to 50 to $\sim 10^{6}$ respectively.

Table 2 Simulation parameter for calculating the effect of changing fixed beam contrast and jitter. The shot-to-shot contrast $\sigma_{T}$ is calculated from the combination of fixed beam contrast and jitter.

\begin{tabular}{lcc}
\hline \hline $\begin{array}{l}\text { Beam contrast, } \\
\sigma_{S}(\%)\end{array}$ & $\begin{array}{c}\text { Beam jitter, } \\
\Delta(\mathrm{mm})\end{array}$ & $\begin{array}{c}\text { Shot-to-shot } \\
\text { contrast, } \sigma_{T}(\%)\end{array}$ \\
\hline 15 & 1 & 5.1 \\
15 & 2 & 6.7 \\
15 & 3 & 8.0 \\
10 & 3 & 5.3 \\
5 & 3 & 2.7 \\
\hline \hline
\end{tabular}



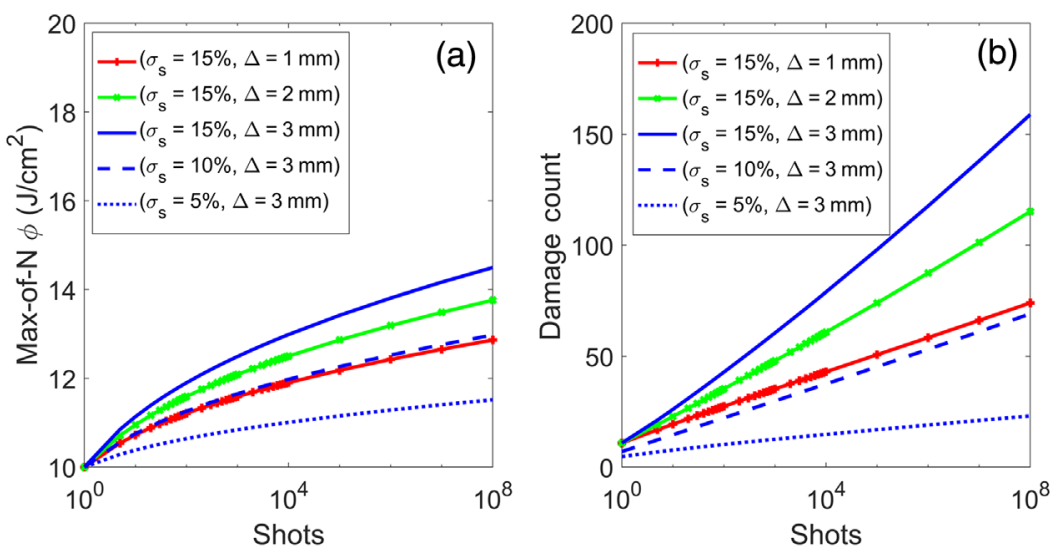

Fig. 12 (a) Calculated Max-of-N mean fluence and (b) damage count versus shots for various mitigation factors, such as reducing beam contrast $\left(\sigma_{s}\right)$ or beam jitter $(\Delta)$.

\section{Conclusion}

We have explored the fundamental mechanism that is responsible for the Max-of-N effect on NIF and have found that it is driven by fixed spatial structure and the slight pointing jitter of the laser. An ensemble statistics model of how jitter in conjunction with fixed spatial contrast impacts the Max-of-N statistic is formulated yielding several improvements over our previously developed ad-hoc model by linking a previously empirically driven fitting parameter (i.e., shot-to-shot contrast $\sigma_{T}$ ) to beam statistics linked to jitter. It also strengthens our understanding of the limit and mitigation of the Max-of-N effect, so we can quantify the impact that different mitigation strategies have on important parameters such as optics lifetime.

\section{Acknowledgments}

The authors thank Benny Evangelista for copy editing this manuscript. This work is performed under the auspices of the U.S. Department of Energy by Lawrence Livermore National Laboratory under Contract No. DE-AC52-07NA27344. (LLNL-JRNL-795163).

\section{References}

1. P. Wegner et al., "NIF final optics system: frequency conversion and beam conditioning," Proc. SPIE 5341, 180-189 (2004).

2. C. A. Haynam et al., "National Ignition Facility laser performance status," Appl. Opt. 46, 3276-3303 (2007).

3. J. Bude et al., "High fluence laser damage precursors and their mitigation in fused silica," Opt. Express 22, 5839-5851 (2014).

4. R. Raman et al., "Laser pre-exposure to mitigate damage on microparticle-contaminated fused silica surface in high power laser systems," Proc. SPIE 10520, 105201T (2018).

5. Z. M. Liao et al., "Contamination-driven laser-induced damage model at the National Ignition Facility," Proc. SPIE 10748, 104780J (2018).

6. A. Melninkaitis et al., "The effect of pseudo-accumulation in the measurement of fatigue laser-induced damage threshold," Proc. SPIE 7132, 713203 (2008).

7. J. Trenholme and M. L. Spaeth, LLNL internal communication (2011).

8. C. W. Carr et al., "Techniques for qualitative and quantitative measurement of aspects of laser-induced damage important for laser beam propagation," Meas. Sci. Technol. 17, 1958-1962 (2006).

9. C. W. Carr et al., "Effect of temporal pulse shape on optical damage," Appl. Phys. Lett. 90, 041110 (2007).

10. Z. M. Liao et al., "Modeling Max-of-N fluence distribution using measured shot-to-shot beam contrast," Appl. Opt. 50, 3547-3552 (2011). 
11. Z. M. Liao et al., "Modeling Max-of-N fluence distribution for optics lifetime," Proc. SPIE, 8190, 81900W (2011).

12. H. A. David and H. N. Nagaraja, Order Statistics, Wiley series in probability and statistics, Wiley Publishing (2003).

13. Wikipedia, "Bapat-Beg Theorem," https://en.wikipedia.org/wiki/Bapat-Beg_theorem (2020).

14. T. I. Suratwala et al., "HF-based etching processes for improving laser damage resistance of fused silica optical surfaces," J. Am. Ceram. Soc. 94, 416-428 (2011).

Zhi M. Liao is a laser physicist at the National Ignition Facility and Photon Science (NIF\&PS) at Lawrence Livermore National Laboratory (LLNL). He joined LLNL in 2001 after graduating from the University of Rochester, where he obtained his BS and MS degrees and his $\mathrm{PhD}$ in optical engineering, working under Dr. Govind Agrawal on nonlinear fiber optics. His expertise is in laser-induced optic damage, nonlinear optics, and adaptive optics. He has contributed to many of LLNL's successful laser projects over the years, such as the fiber laser guide star, alkali laser, the mercury laser, and NIF. He has authored over 60 publications covering experimental and modeling results and has given many talks on the topic as an invited speaker and guest lecturer. He has three patents and was the co-PI for the team that won an R\&D award for highaverage-power frequency conversion using YCOB crystal. Currently, he is the leading researcher for modeling optic damage and lifetime for NIF. He is a member of SPIE and a senior member of OSA and has served as an executive committee member (including chair) of OSA technical groups. He also has served on the international program committee for the Pacific Rim Laser Damage conference since 2016.

Giuliana Pallotta is a statistician working in the Applied Statistics Group at Lawrence Livermore National Laboratory (LLNL) Computational Engineering Division since 2015. Prior to joining LLNL as a staff member, she was a research scientist in maritime situational awareness at NATO Science and Technology Organization CMRE. She is a Fulbright Scholar. Her research interests are in the areas of Bayesian inference, statistical modeling, machine learning, anomaly detection, reliability, and information fusion for surveillance and security.

Biographies of the other authors are not available. 